\title{
DIMENSÕES DAS FIBRAS E SUA RELAÇÃO COM A IDADE DE TRANSIÇÃO ENTRE LENHO JUVENIL E ADULTO DE Eucalyptus grandis W. Hill ex Maiden
}

\author{
FIBER DIMENSIONS AND THEIR RELATIONSHIP WITH THE TRANSITION AGE BETWEEN \\ JUVENILE AND MATURE WOOD OF Eucalyptus grandis W. Hill ex Maiden
}

\author{
Rômulo Trevisan ${ }^{1}$ Magda Rosa ${ }^{2}$ Clovis Roberto Haselein ${ }^{3}$ Elio José Santini ${ }^{4}$ \\ Darci Alberto Gatto ${ }^{5}$
}

\begin{abstract}
RESUMO
Este estudo teve como objetivo avaliar as dimensões das fibras e a idade de transição entre lenho juvenil e adulto da madeira de Eucalyptus grandis. Para tanto, foram amostradas, com base no diâmetro dominante de Assmann, quatro árvores de um povoamento experimental situado próximo ao litoral do Rio Grande do Sul com 18 anos de idade. Após o abate, no disco correspondente à região do DAP (diâmetro à altura do peito) foram marcadas e seccionadas baguetas centrais das quais se confeccionaram fragmentos selecionados em intervalos regulares, no sentido medula-casca, para maceração e, posteriormente, determinação do comprimento, do diâmetro total, do diâmetro do lume e da espessura da parede das fibras com auxílio de um microscópio ótico com ocular graduada. A segregação dos dois tipos de lenho foi determinada pela variação radial do comprimento das fibras utilizando regressões lineares múltiplas e simples; e a idade de transição por meio de regressões lineares simples. Os menores valores referentes às dimensões de comprimento, de diâmetro total e da espessura da parede das fibras foram observados próximo à medula, com tendência crescente em direção à casca, sugerindo a existência de diferenças entre os lenhos próximo da medula e da casca. Entretanto, esse padrão não foi observado para o diâmetro do lume das fibras o qual evidenciou um aumento até a posição central do raio seguido de diminuição até a porção mais externa. A zona de transição entre lenho juvenil e adulto foi estabelecida entre as idades de 4,2 e 4,7 até 8,1 e 9,1 anos, sendo denominada de faixa de lenho juvenil aquela correspondente até 4,2 e 4,7 anos; e de lenho adulto superior a 8,1 e 9,1 anos. Já a transição entre o lenho juvenil e adulto ocorreu a $10 \mathrm{~cm}$ da medula, ou seja, na idade de, aproximadamente, 6,1 anos. A definição da idade em que ocorre a transição entre os lenhos juvenil e adulto facilita os processos de transformação da madeira uma vez que a porção juvenil constitui um obstáculo à viabilização do seu uso industrial.
\end{abstract}

Palavras-chave: elementos anatômicos; segregação dos lenhos; qualidade da madeira.

1 Engenheiro Florestal, Dr., Professor Adjunto do Departamento de Engenharia Florestal, Universidade Federal de Santa Maria Campus Frederico Westphalen, BR386 Km40, Linha 7 de Setembro, CEP 98400-000, Frederico Westphalen (RS), Brasil.romulo_trevisan@ufsm.br

2 Engenheira Florestal, Mestranda em Agronomia, Programa de Pós-Graduação em Agronomia: Agricultura e Ambiente, Universidade Federal de Santa Maria Campus Frederico Westphalen, BR386 Km40, Linha 7 de Setembro, CEP 98400-000, Frederico Westphalen (RS), Brasil. magda-ros@hotmail.com

3 Engenheiro Florestal, PhD., Professor titular do Departamento de Ciências Florestais, Centro de Ciências Rurais, Universidade Federal de Santa Maria, Av. Roraima, 1000, Bairro Camobi, CEP 97105-900, Santa Maria (RS), Brasil. clovis.haselein@ufsm.br

4 Engenheiro Florestal, Dr., Professor Titular do Departamento de Ciências Florestais, Centro de Ciências Rurais, Universidade Federal de Santa Maria, Av. Roraima, 1000, Bairro Camobi, CEP 97105-900, Santa Maria (RS), Brasil.santini@ufsm.br

5 Engenheiro Florestal, Dr., Professor Adjunto da Engenharia Industrial Madeireira, Centro de Engenharias, Universidade Federal de Pelotas, Rua Conde de Porto Alegre, 793, CEP 96010-290, Pelotas (RS), Brasil. darcigatto@yahoo.com 


\begin{abstract}
This study was undertaken to evaluate the fiber dimensions and the transition age between juvenile and mature wood of Eucalyptus grandis. It was selected, based on the Assmann's dominant diameter, four trees of an experimental stand located in the northern coast of Rio Grande do Sul state, with 18 years of age. After cutting down, in the disk corresponding to the region of the DBH (diameter at breast height) were marked and sectioned central baguettes, where crafted fragments were selected at regular intervals in pith-to-bark direction for maceration and, thereafter, determination of the length, the total diameter, the lume diameter and the wall thickness of the fibers with the aid of an optical microscope with a graduated eyepiece. The segregation of both types of wood was determined by the radial variation of the fiber length and using simple and multiple linear regressions; and a transitional age through simple linear regression. The lower values for the dimensions of length, total diameter and wall thickness of the fibers were observed near the pith, with an increasing trend toward the bark, suggesting the existence of differences between wood near the pith and the bark. However, this standard was not observed in the lume diameter of the fibers, which showed an increase to the middle position of the radius followed by a decrease to the outer portion. The transition zone between juvenile and mature wood has been established between the ages of 4.2 and 4.7 up to 8.1 and 9.1 years, range termed juvenile wood that corresponding to 4.2 and 4.7 years; and adult wood exceeding 8.1 and 9.1 years. The transition between adult and juvenile wood occurred at $10 \mathrm{~cm}$ from the pith, that is, in the age of about 6.1 years. The definition of the age at which the transition between the juvenile and mature wood occurs facilitates the wood transformation processes once the juvenile portion constitutes an obstacle to the viability of its industrial use.
\end{abstract}

Keywords: anatomical elements; segregation of wood; wood quality.

\title{
INTRODUÇÃO
}

Nas últimas décadas, a matriz produtiva do setor florestal brasileiro, em virtude do constante aprimoramento de técnicas para produção de madeira em quantidade e com qualidade, da pressão exercida pela sociedade e, fundamentalmente, por almejar um desenvolvimento sustentável, tem apresentado uma tendência exponencial de substituição da matéria-prima oriunda de espécies nativas por produtos provenientes de reflorestamentos de rápido crescimento e alta produtividade (BALLARIN; PALMA, 2003; PALMA; LEONELLO; BALLARIN, 2010; SETTE JUNIOR et al., 2012).

Paralelo a esse propósito, há um consenso da dificuldade de produção de madeira com qualidade, pois esse material é originário de um sistema biológico complexo e suas propriedades físico-mecânicas variam entre espécies, entre árvores de uma mesma espécie e nas diferentes partes de uma mesma árvore, como no sentido medula-casca ou mesmo nos anéis de crescimento (GONÇALVES et al., 2007), em função de fatores ambientais e genéticos, bem como das suas interações (TREVISAN et al., 2013), fundamentando sua completa caracterização (SETTE JUNIOR et al., 2012).

De modo geral, a variação radial, objeto dessa análise, se dá em função da presença do lenho juvenil, ou seja, xilema secundário formado durante a fase jovem do câmbio vascular, sua proporção no tronco, além de suas características físico-químicas e anatômicas (GONÇALVES et al., 2007; LEONELLO; PALMA; BALLARIN, 2008; GATTO et al., 2010; RAMOS et al., 2011). A madeira juvenil se caracteriza por apresentar menor massa específica, maior ângulo das microfibrilas na camada S2 da parede celular, fibras mais curtas, menor contração transversal e maior longitudinal, maior volume de lenho de reação, menor proporção de lenho tardio, paredes celulares mais finas e menor resistência mecânica em relação à madeira adulta, a qual possui propriedades mais preferíveis em função da qualidade (BENDTSEN, 1978; ZOBEL, 1984; SENFT; BENDTESEN; GALLIGAN, 1985; LARSON et al., 2001; NIGOSKI, 2005; LEONELLO; PALMA; BALLARIN, 2008).

A proporção de madeira juvenil existente em uma tora é uma característica que depende da idade da árvore, do ambiente e do manejo empregado (PALMA; LEONELLO; BALLARIN, 2010); porém, cabe ressaltar que, em certos casos, com tratos silviculturais semelhantes, verifica-se uma expressiva variabilidade em decorrência da desproporcionalidade entre os lenhos juvenil e adulto acumulados no tronco 
(LEONELLO; PALMA; BALLARIN, 2008). Desse modo, fica perceptível a importância do conhecimento das características anatômicas desse material como, por exemplo, as dimensões dos vasos e das fibras, as quais desempenham papel fundamental em processos fisiológicos e de sustentação.

Assim, a diferença fundamental entre o lenho juvenil e adulto se dá pela variação das propriedades anatômicas da madeira (GATTO et al., 2008; RAMOS et al., 2011), na qual a porção juvenil caracteriza-se por menores valores das dimensões das células e por alterações na sua forma, estrutura e disposição em sucessivos anéis de crescimento, além de uma menor massa específica (RAMAY; BRIGGS, 1986; GATTO et al., 2010); possibilitando a determinação da segregação dos lenhos em função dos caracteres anatômicos e/ou da variação radial da massa específica.

A determinação da idade de transição entre lenhos em uma espécie florestal é de suma importância para a qualidade do material obtido, uma vez que a madeira adulta apresenta melhores propriedades físicas e mecânicas (BENDTSEN, SENFT, 1986; GATTO et al., 2007). Dessa forma, o presente estudo teve por objetivo avaliar as dimensões das fibras e a idade de transição entre lenho juvenil e adulto da madeira de Eucalyptus grandis W. Hill ex Maiden, aos 18 anos de idade, bem como a variação radial desses elementos anatômicos visando à utilização adequada e com qualidade do material.

\section{MATERIAL E MÉTODO}

O material utilizado no estudo foi proveniente de um povoamento experimental de Eucalyptus grandis W. Hill ex Maiden pertencente à Empresa Flosul Indústria e Comércio de Madeiras Ltda., localizada no município de Capivari do Sul, litoral norte do Estado do Rio Grande do Sul. O povoamento, situado, aproximadamente, nas coordenadas geográficas de 50³0' de longitude Oeste e $30^{\circ} 08^{\prime}$ de latitude Sul, foi implantado em maio de 1990 com espaçamento inicial de 3,0 x 1,7 m e não foi submetido a tratamentos silviculturais.

As árvores amostradas aos 18 anos de idade, com diâmetro médio igual a 34,2 $\pm 2,0 \mathrm{~cm}$ e altura média de 40,9 $\pm 1,3 \mathrm{~m}$, foram selecionadas utilizando o conceito de Assmann, o qual emprega a mensuração dos 100 exemplares de maior diâmetro por hectare. Dessa forma, com auxílio de uma regra de três simples e considerando-se a área interna de cada parcela foi determinado o número de indivíduos dominantes e, em seguida, calculada a árvore dominante média a ser amostrada $\left(\mathrm{d}_{100}\right)$. Para o estudo das dimensões das fibras e da idade de transição dos lenhos, no sentido medula-casca, foi realizada a extração de um disco do tronco, situado na altura de 1,30 m (diâmetro à altura do peito), de cada uma das quatro árvores selecionadas.

Em seguida os discos foram identificados de acordo com a localização experimental, embalados em sacos plásticos e transportados para o Laboratório de Produtos Florestais (LPF) da Universidade Federal de Santa Maria (UFSM), onde foram marcados e seccionados em baguetas centrais (contendo a medula) de $2 \mathrm{~cm}$ de largura, sendo selecionada para maceração apenas a porção livre de defeitos. O material restante foi armazenado em câmara climatizada a uma temperatura de $20^{\circ} \mathrm{C}$ e umidade relativa do ar igual a $65 \%$, conservando suas propriedades em razão de uma eventual substituição.

As amostras destinadas à maceração foram novamente seccionadas a cada centímetro ao longo de uma linha reta no sentido medula-casca, ao passo que os fragmentos resultantes dessa atividade foram devidamente identificados e acondicionados em sacos de papel na câmara climatizada. Para a maceração, foram selecionados os fragmentos com intervalos regulares de $2 \mathrm{~cm}$ e, com o uso de um estilete, confeccionadas lascas de madeira semelhantes a palitos de fósforo, porém, mais estreitas, as quais foram alocadas em tubos de ensaio numerados. A dissociação do tecido lenhoso foi realizada pelo método de peróxido de hidrogênio e ácido acético, conforme descrito por Kraus e Arduin (1997).

Após essa etapa, oproduto da maceração resultou emuma pasta de fibras com coloração esbranquiçada, a qual foi disposta em um papel-filtro sobre um funil, lavada com água destilada e, em seguida, com etanol $50 \%$. Depois de concluídos esses procedimentos, o processo foi finalizado com a coloração das fibras em solução aquosa de safranina 1\%, durante trinta minutos. Em seguida, foi efetuada, para cada fragmento amostrado, a montagem de três lâminas semipermanentes, utilizadas para a determinação de 30 leituras do comprimento, do diâmetro total, do diâmetro do lume e da espessura das fibras em microscópio ótico com ocular graduada, conforme as normas do IAWA - Committee (INTERNATIONAL ASSOCIATION OF 


\section{WOOD ANATOMY, 1989).}

Os dados das dimensões das fibras das árvores dominantes foram agrupados em três posições equidistantes, no sentido medula-casca, denominadas de: posição interna, correspondente ao primeiro terço do raio; central, igual ao segundo terço e posição externa, pertencente ao terceiro terço. Estes dados foram submetidos à análise de variância para delineamento experimental inteiramente casualizado e, no caso de rejeição da hipótese de igualdade de médias, ao teste T - DMS (Diferença Mínima Significativa, $\alpha=5 \%$ ).

Após a constatação da presença de lenho adulto, definido pelo teste de médias entre as posições, a determinação da transição entre os lenhos foi realizada, primeiramente, com o auxílio da metodologia descrita por Ramos et al. (2011) na qual são utilizadas regressões lineares múltipla e simples. Dessa forma, os dados de comprimento de fibras amostrados no sentido medula-casca foram ajustados à variação radial utilizando uma regressão linear múltipla resultando em dois pontos de inflexão que foram utilizados para delimitação da zona de segregação entre os lenhos juvenil e adulto. A partir dessa análise, entre as inflexões encontradas, foi realizada uma regressão linear simples tangenciando a curva dos dados estimados para realçar o início e o final dessa faixa. As posições radiais resultantes foram comparadas com os dados médios dos inventários realizados nas quatro árvores amostradas (Tabela 1) no intuito de estimar a zona de transição, bem como os lenhos juvenil e adulto.

TABELA 1: Dados médios dos inventários realizados nas quatro árvores dominantes amostradas de Eucalyptus grandis.

TABLE 1: Average data on inventories conducted in the four sampled dominant trees of Eucalyptus grandis.

\begin{tabular}{|c|c|c|c|c|c|c|c|c|c|c|c|c|c|c|}
\hline $\begin{array}{l}\text { Idade } \\
\text { (anos) }\end{array}$ & 4,2 & 4,7 & 5,1 & 6,1 & 7,1 & 8,1 & 9,1 & 10,7 & 11,7 & 12,7 & 14,2 & 14,7 & 15,7 & 16,5 \\
\hline $\mathrm{d}_{100}$ & 15,5 & 17,3 & 18,4 & 20,6 & 22,2 & 23,3 & 26,2 & 27,1 & 28,4 & 29,1 & 31,0 & 31,4 & 31,8 & 32,7 \\
\hline$(\boldsymbol{\sigma})$ & $( \pm 0,49)$ & $( \pm 0,60)$ & $( \pm 0,64)$ & $( \pm 0,89)$ & $( \pm 1,18)$ & $( \pm 1,73)$ & $( \pm 1,18)$ & $( \pm 1,38)$ & $( \pm 1,27)$ & $( \pm 1,20)$ & $( \pm 1,36)$ & $( \pm 1,60)$ & $( \pm 1,68)$ & $( \pm 1,66)$ \\
\hline $\mathrm{h}_{100}$ & 17,7 & 20,5 & 22,0 & 25,0 & 25,2 & 28,9 & 29,3 & 30,5 & 31,6 & 32,6 & 33,4 & 33,0 & 34,2 & 37,4 \\
\hline$(\boldsymbol{\sigma})$ & $( \pm 1,93)$ & $( \pm 0,58)$ & $( \pm 0,58)$ & $( \pm 1,08)$ & $( \pm 0,87)$ & $( \pm 0,48)$ & $( \pm 1,32)$ & $( \pm 1,73)$ & $( \pm 1,65)$ & $( \pm 1,31)$ & $( \pm 1,25)$ & $( \pm 1,11)$ & $( \pm 0,50)$ & $( \pm 1,93)$ \\
\hline
\end{tabular}

Em que: $\mathrm{d}_{100}=$ diâmetro da árvore dominante de Assmann, em $\mathrm{cm} ; \mathrm{h}_{100}=$ altura da árvore dominante de Assmann, em m; $\sigma=$ desvio padrão da média, em cm para $\mathrm{d}_{100} \mathrm{e} \mathrm{m}_{\text {para }} \mathrm{h}_{100}$.

A idade de transição dos lenhos foi definida por meio de duas regressões lineares simples, uma aplicada para a parte ascendente da distribuição radial (medula-casca) do comprimento das fibras e outra para a parte em que a mesma se mostrava constante ou descendente. Os limites das duas distribuições de pontos amostrais foram definidos visualmente em gráfico antes do ajuste das equações. A posição radial de início de formação de lenho adulto foi determinada pelo cruzamento das duas retas obtidas pelas equações de regressão (GATTO et al., 2007) e comparada com os dados médios dos inventários realizados nas quatro árvores amostradas (Tabela 1) no intuito de estimar a idade de transição.

\section{RESULTADOS E DISCUSSÃO}

As dimensões médias das fibras, descritas na Tabela 2, situaram-se nas faixas de variação estabelecidas pela literatura para a variável comprimento, com valores de 750 a $1520 \mu \mathrm{m}$ (BARRICHELO; BRITO, 1976; TOMAZELLO FILHO, 1985; SILVA, 2002; ROCHA; FLORSHEIM; COUTO, 2004; CALONEGO; SEVERO; ASSIS, 2005; GOMIDE et al., 2005; VEENIN et al., 2005; SILVA et al., 2007; LEONELLO; PALMA; BALLARIN, 2008; GATTO et al., 2010; PALMA; LEONELLO; BALLARIN, 2010; RAMOS et al., 2011; SETTE JUNIOR et al., 2012; TREVISAN, et al., 2013); e espessura da parede da fibra com valores entre 2,5 a 6,0 $\mu \mathrm{m}$ (TOMAZELLO FILHO, 1985; ROCHA; FLORSHEIM; COUTO, 2004; GOMIDE et al., 2005; SILVA et al., 2007; RAMOS et al., 2011; SETTE JUNIOR et al., 2012). 
TABELA 2: Valores médios do comprimento, do diâmetro do lume, do diâmetro total e da espessura da parede das fibras para as árvores dominantes de Eucalyptus grandis e análise de variância dessas variáveis em função da posição radial no sentido medula-casca.

TABLE 2: Mean values for length, lume diameter, the total diameter and wall thickness of the fibers to the dominant trees of Eucalyptus grandis and analysis of variance of these variables as a function of radial position in the pith-bark direction.

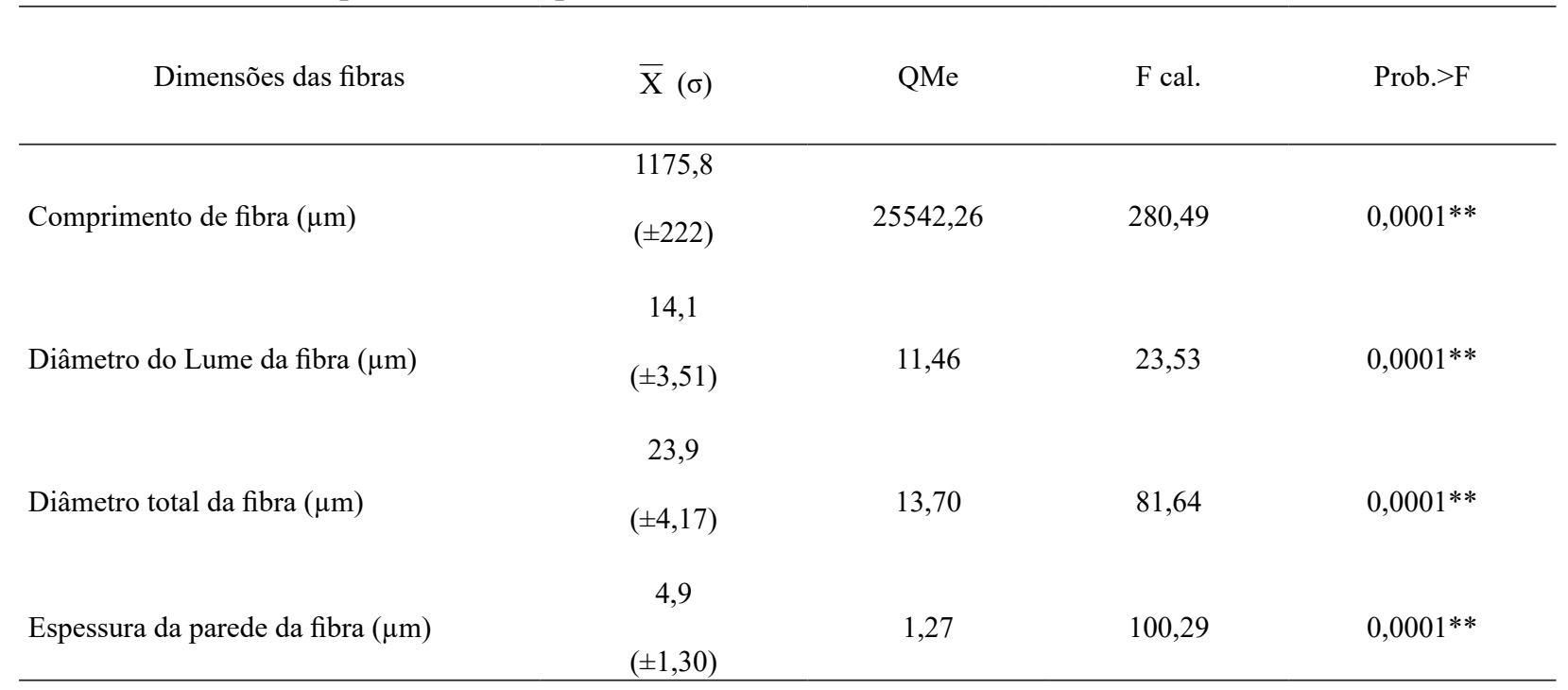

Em que: ${ }^{\bar{x}}=$ média da variável analisada, em $\mu \mathrm{m} ; \sigma=$ Desvio padrão da média, em $\mu \mathrm{m} ; \mathrm{QMe}=$ Quadrado médio do erro; Fcal. $=$ Valor de $\mathrm{F}$ calculado; Prob. $>\mathrm{F}=$ nível de probabilidade de erro; $* *=$ significativo a $1 \%$ de probabilidade de erro.

Entretanto, mesmo as variáveis diâmetro do lume e diâmetro total da fibra, com médias de 14,1 $\mu \mathrm{m}$ e $23,9 \mu \mathrm{m}$, respectivamente, apesar de ligeiramente superiores aos resultados normalmente reportados pela literatura, com valores entre 6 a $12 \mu \mathrm{m}$ e de 12 a $20 \mu \mathrm{m}$, respectivamente (ROCHA; FLORSHEIM; COUTO, 2004; GOMIDE et al., 2005; SILVA et al., 2007), foram consideradas normais e semelhantes às descritas por Ramos et al. (2011).

A análise de variância evidenciou diferenças em função da posição no sentido medula-casca para todas as dimensões das fibras avaliadas, inclusive para o comprimento, característica comumente utilizada para definição da idade de transição (GATTO et al., 2007; 2010), constatando uma provável existência de

TABELA 3: Análise de variância das dimensões das fibras da madeira das árvores dominantes de Eucalyptus grandis em função da posição no sentido medula-casca.

TABLE 3: Analysis of variance of fiber dimensions of wood from dominant trees of Eucalyptus grandis due to the position in the pith-bark direction.

\begin{tabular}{lcc}
\hline \multicolumn{1}{c}{ Dimensões das fibras } & \multicolumn{2}{c}{ Posições amostradas no sentido medula-casca } \\
\cline { 2 - 3 } & Interna $(\mathrm{CV})$ & Central $(\mathrm{CV})$ \\
\hline Comprimento de fibra $(\mu \mathrm{m})$ & $960,33 \mathrm{c}(16,0)$ & $1234,75 \mathrm{~b}(13,2)$ \\
Diâmetro do Lume da fibra $(\mu \mathrm{m})$ & $13,20 \mathrm{c}(23,1)$ & $15,30 \mathrm{a}(21,6)$ \\
Diâmetro total da fibra $(\mu \mathrm{m})$ & $21,20 \mathrm{~b}(16,6)$ & $24,94 \mathrm{a}(14,4)$ \\
Espessura da parede da fibra $(\mu \mathrm{m})$ & $4,00 \mathrm{c}(25,5)$ & $4,82 \mathrm{~b}(23,2)$
\end{tabular}

Em que: $\mathrm{CV}=$ Coeficiente de variação, em \%; Médias seguidas de mesma letra na linha não diferem estatisticamente pelo teste DMS (Diferença Mínima Significativa) com confiabilidade de 95\%. 
diferentes tipos de lenho no material amostrado. Além dessa verificação, na Tabela 3 pode ser observado que apenas para o diâmetro do lume da fibra os resultados não mostraram uma tendência de variação crescente na direção radial.

A tendência crescente do comprimento de fibra médio por posição amostrada no sentido medulacasca já era esperada e semelhante aos resultados encontrados por Malan e Gerischer (1987), Bhat, Bhat e Dhamodaran (1990), Shimoyama (1990), Malan e Hoon (1992), Cruz (2000), Silva (2001; 2002), Rocha, Florsheim e Couto (2004), Palma, Leonello e Ballarin (2010), Ramos et al. (2011), Sette Junior et al. (2012) e Trevisan et al. (2013). Além disso, o coeficiente de variação dessa variável diminuiu quanto mais próximo da casca indicando maior homogeneidade nos comprimentos das fibras na região externa, da mesma forma que os citados por Leonello, Palma e Ballarin (2008), Gatto et al. (2010) e Palma, Leonello e Ballarin (2010).

Esses resultados estão em conformidade com os relatados por Tomazello Filho (1987), nos quais o autor refere que a estabilização do comprimento, para várias espécies de eucalipto, ocorre quando as células do câmbio atingem comprimento máximo, iniciando a formação do lenho adulto. A variabilidade do comprimento das fibras no sentido radial deve-se, provavelmente, à presença de uma zona de madeira juvenil próxima à medula, a qual apresenta, dentre outras características, fibras curtas, com menor diâmetro, paredes mais estreitas e maior ângulo fibrilar na camada S2, o que repercute diretamente na qualidade da madeira (TOMAZELLO FILHO, 1987; SETTE JUNIOR et al., 2012; TREVISAN et al., 2013).

Outra característica que apresentou variação radial com valores médios estatisticamente diferentes e aumentando na direção da casca (Tabela 3) foi a espessura da parede das fibras sugerindo a existência de diferenças entre os lenhos próximo da medula e da casca. Da mesma forma, no entanto, com diferenças apenas na posição interna, verificou-se uma tendência de aumento do diâmetro total da fibra. Essas variações crescentes, tanto para a espessura quanto para o diâmetro total, também foram reportadas por autores como Rocha, Florsheim e Couto (2004), Silva et al. (2007) e Sette Junior et al. (2012).

Uma explicação para essa variação seria de que as mudanças nas dimensões dos elementos anatômicos do lenho ocorrem em função do envelhecimento das células do câmbio vascular resultando em modificações fisiológicas e moleculares (PLOMION; LEPROVOST; STOKES, 2001). Assim, no xilema primário da zona cambial, as divisões celulares são menos frequentes com o aumento da idade da árvore, permitindo que os elementos vasculares tenham mais tempo para sua elongação longitudinal e transversal, passando a formar estruturas de maiores dimensões até atingir a estabilização em idades mais avançadas (TOMAZELLO FILHO, 1985).

A única característica das dimensões das fibras em que os resultados não mostraram variação nítida no sentido medula-casca foi o diâmetro do lume, o qual apresentou um acréscimo até a posição central amostrada com posterior decréscimo nos valores radiais, todos diferentes estatisticamente. Resultados semelhantes relatados por Ramos et al. (2011) também evidenciaram um aumento até a posição central do raio seguido de diminuição até a porção mais externa, contudo, com valores não estatisticamente diferentes, os quais ressaltaram que o decréscimo no diâmetro do lume pode ser explicado pelo aumento da espessura de parede das fibras.

A partir dos resultados encontrados no teste de médias para comprimento de fibras os dados foram ajustados, após a análise do padrão de distribuição, em uma regressão linear quadrática para verificação da variação em função da posição radial com valor de $\mathrm{F}$ calculado igual a 78,3 em nível de $\alpha=1 \%$ de probabilidade de erro. O ajuste resultou em dois pontos de inflexão, os quais foram utilizados com auxílio de uma regressão linear simples que apresentou valor de F calculado de 12,0 e mesmo nível de probabilidade de erro da anterior, para delimitação da zona de segregação entre os lenhos juvenil e adulto (Figura 1).

As análises demonstradas na Figura 1 evidenciaram um aumento acentuado no comprimento das fibras até, aproximadamente, $8 \mathrm{~cm}$ da medula. A partir desse ponto, ocorreu uma tendência de diminuição nos valores, entre 8 a cerca de 12 e $13 \mathrm{~cm}$ e, de estabilização após essa faixa quanto mais próximo da casca. Com a definição dessas três regiões, as posições foram transformadas em diâmetro e comparadas com os dados médios de inventário das árvores amostradas (Tabela 1) sendo estabelecidas as faixas de lenho juvenil (até 4,2 e 4,7 anos); de transição entre lenho juvenil e adulto (de 4,2 e 4,7 até 8,1 e 9,1 anos); e de lenho adulto (superior a 8,1 e 9,1 anos).

Resultados semelhantes foram descritos por Ramos et al. (2011) que, estudando a madeira de 


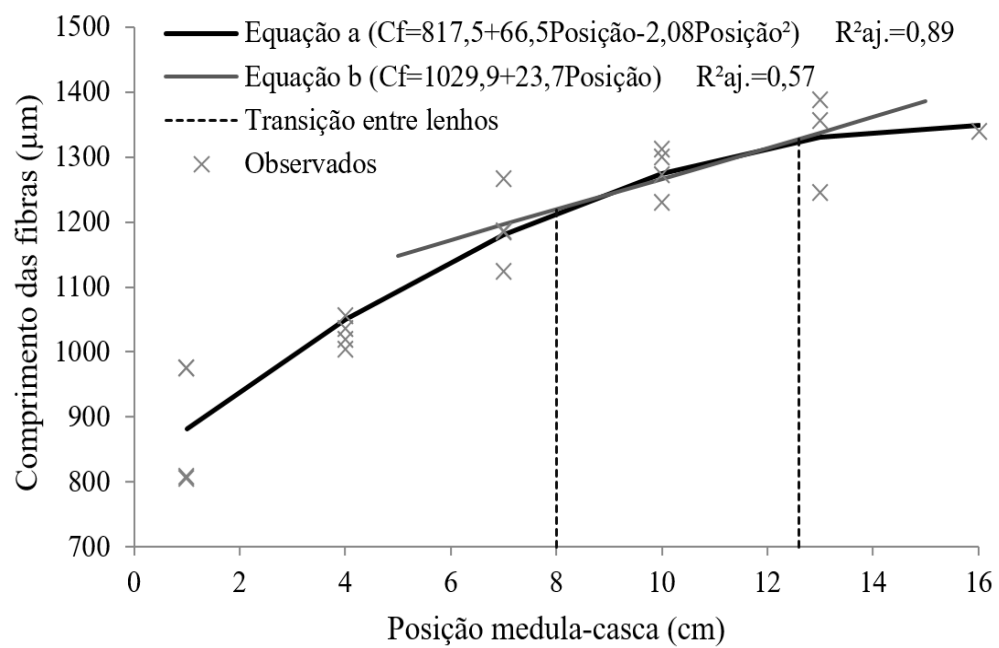

FIGURA 1: Delimitação da zona de segregação entre os lenhos juvenil e adulto de Eucalyptus grandis em função da posição no sentido medula-casca.

FIGURE 1: Delimitation of the segregation zone between juvenile and mature woods of due to the position in the pith-bark direction.

Eucalyptus grandis, aos 23 anos de idade, estabeleceram que a zona de transição do lenho juvenil e adulto ocorreu entre o $5^{\circ}$ e $11^{\circ}$ ano tanto para as análises em função do comprimento de fibras quanto aquelas que utilizaram o ângulo microfibrilar. Da mesma forma, Palma, Leonello e Ballarin (2010) citaram que a região de madeira juvenil para Corymbia citriodora, aos 29 anos de idade, compreende a faixa até, aproximadamente, $11 \mathrm{~cm}$ da medula e, a madeira adulta tem início em $11 \mathrm{e} 12 \mathrm{~cm}$ com extensão até a casca, mesmo quando as análises foram realizadas em cada uma das árvores.

No intuito de estabelecer a idade de transição entre os lenhos, os dados de comprimento de fibras no sentido medula-casca foram ajustados por duas regressões lineares simples denominadas de Equação a (com valor de $\mathrm{F}$ calculado igual a 110,8 em nível de $\alpha=1 \%$ de probabilidade de erro) e Equação $\mathrm{b}$ (com valor de F calculado igual a 12,9 e mesmo nível de probabilidade de erro), conforme demonstrado na Figura 2.

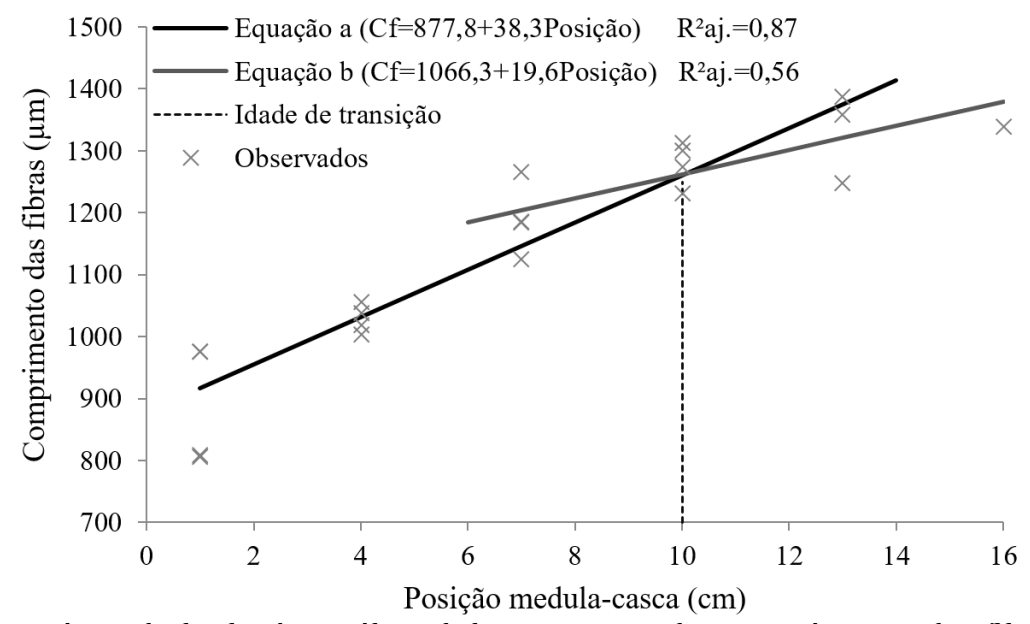

FIGURA 2: Idade de transição do lenho juvenil e adulto em razão do comprimento das fibras da madeira de Eucalyptus grandis no sentido medula-casca.

FIGURE 2: Age of transition from juvenile and mature wood due to the length of fibers of wood from Eucalyptus grandis in the pith-bark direction. 
O resultado evidenciado na Figura 2 permitiu a definição estimada da transição dos lenhos a partir de $10 \mathrm{~cm}$ da medula e, após a comparação com os dados de diâmetro dominante médio dos inventários realizados nas quatro árvores amostradas (Tabela 1), na idade de, aproximadamente, 6,1 anos.

Dessa forma, considerando aspectos práticos, a definição da idade em que ocorre a transição entre os lenhos juvenil e adulto facilita os processos de transformação da madeira, uma vez que a porção juvenil constitui um obstáculo à viabilização do seu uso industrial.

\section{CONCLUSÕES}

As variações das dimensões de comprimento, de diâmetro total e da espessura das fibras apresentaram uma tendência crescente no sentido radial sugerindo a existência de diferenças entre os lenhos próximo da medula e da casca. De outra forma, esse padrão não foi observado para o diâmetro do lume das fibras, o qual evidenciou um aumento até a posição central do raio seguido de diminuição até a porção mais externa.

A zona de transição entre lenho juvenil e adulto foi estabelecida entre as idades de 4,2 até 9,1 anos. Já a transição entre o lenho juvenil e adulto ocorreu na idade de, aproximadamente, 6,1 anos.

\section{REFERÊNCIAS}

BALLARIN A. W.; PALMA, H. A. L. Propriedades da resistência e rigidez da madeira juvenil e adulta de Pinus taeda L. Revista Árvore, Viçosa, MG, v. 27, n. 3, p. 371-380, 2003.

BARRICHELO, L. E. G.; BRITO, J. O. A madeira das espécies de eucalipto como matéria prima para a indústria de celulose e papel. PRODEPEF - Projeto de desenvolvimento e pesquisa florestal, Brasília, n. 13, p. 1-145, 1976.

BENDTSEN B. A. Properties of wood from improved and intensively managed trees. Forest Products Journal, Peachtree Corners, v. 28, n. 10, p. 61-71, 1978.

BENDTSEN B. A.; SENFT, J. Mechanical and anatomical properties in individual growth rings of plantation-grown cottonwood and loblolly pine. Wood and Fiber Science, Madison, v. 18, n. 1, p. 23-28, 1986.

BHAT, K. M.; BHAT, K. V.; DHAMODARAN, T. K. Wood density and fibre length of Eucalyptus grandis grown in Kerala, India. Wood and Fiber Science, Madison, v. 22, n. 1, p. 54-61, 1990.

CALONEGO, F. W.; SEVERO, E. T. D.; ASSIS, P. P. Mensuração do comprimento das fibras para determinação da madeira juvenil em Eucalyptus citriodora. Scientia Forestalis, Piracicaba, n. 68, p. 113-121, 2005.

CRUZ, C. R. Caracterização da madeira de clones de Eucalyptus para a utilização na indústria madeireira. 2000. 64 f. Dissertação (Mestrado em Engenharia Florestal) - Universidade Federal do Paraná, Curitiba, 2000.

GATTO, D. A. et al. Estimativa da idade de segregação do lenho juvenil e adulto de Carya illinoinensis (Wangenh) K. Koch por meio de parâmetros anatômicos da madeira. Ciência Florestal, Santa Maria, v. 20, n. 4, p. 675-682, 2010.

GATTO, D. A. et al. Estimativa da idade de segregação do lenho juvenil e adulto para Platanus x acerifolia (Ait.) Willd. Cerne, Lavras, v. 13 n. 4, p. 393-398, 2007.

GATTO D. A. et al. Estimativa da idade de segregação do lenho juvenil e adulto por meio de parâmetros anatômicos para madeira de Luehea divaricata Mart. Ciência Florestal, Santa Maria, v. 18, n. 4, p. 535-540, 2008.

GOMIDE, J. L. et al. Caracterização tecnológica, para produção de celulose, da nova geração de clones de Eucalyptus do Brasil. Revista Árvore, Viçosa, MG, v. 29, n. 1, p. 129-137, 2005.

GONÇALVES, M. P. M. et al. Variação radial da densidade básica e comprimento das fibras da madeira de Tectona grandis L. Floresta e Ambiente, Rio de Janeiro, v. 14, n. 1, p. 70-75, 2007.

INTERNATIONAL ASSOCIATION OF WOOD ANATOMY. List of microscopic features for hardwood identification. IAWA Bulletin, Utrecht, v. 10, n. 3, p. 226-332, 1989.

KRAUS, J. E.; ARDUIN, M. Manual básico de métodos em morfologia vegetal. Seropédica: EDUR, 1997. $198 \mathrm{p}$. 
LARSON P. R. et al. Formation and properties of juvenile wood in southern pines. Madison: Forest service, 2001. $46 \mathrm{p}$.

LEONELlO, E. C.; PALMA, H. A. L.; BALLARIN, A. W. Delimitação da madeira juvenil e adulta de Eucalyptus grandis. Revista Forestal Venezolana, Merida, v. 52, n. 1, p. 93-98, 2008.

MALAN, F. S.; GERISCHER, G. F. R. Wood property differences in South African grown Eucalyptus grandis trees of different growth stress levels. Holzforschung, Berlin, v. 41, n. 6, p. 331-335, 1987.

MALAN, F. S.; HOON, M. Effect of initial spacing and thinning on some wood properties of Eucalyptus grandis. South African Forestry Journal, Pretoria, n. 163, p. 13-20, 1992.

NIGOSKI, S. Espectroscopia no infravermelho no estudo de características da madeira e papel de Pinus taeda L. 2005. 160 f. Tese (Doutorado em Ciências Florestais) - Universidade Federal do Paraná, Curitiba, 2005.

PALMA, H. A. L.; LEONELlO, E. C.; BALLARIN, A. W. Demarcação da madeira juvenil e adulta de Corymbia citriodora. Cerne, Lavras, v. 16, supl, p. 141-148, 2010.

PLOMION, C.; LeProvost, G.; STOKES, A. Wood Formation in Trees. Plant Physiology, Bethesda, v. 127, n. 4, p. 1513-1523, 2001.

RAMAY, W.; BRIGGS, D. Juvenile Wood: has it come of age? In: A TECHNICAL workshop: juvenile wood - what does mean to forest management and forest products. Madison: Forest Products Society, 1986. p. $5-11$.

RAMOS, L. M. A. et al. Variação radial dos caracteres anatômicos da madeira de Eucalyptus grandis W. Hill ex Maiden e idade de transição entre lenho juvenil e adulto. Scientia Forestalis, Piracicaba, v. 39, n. 92, p. 411-418, 2011.

ROCHA, F. T.; FLORSHEIM, S. M. B.; COUTO, H. T. Z. Variação das dimensões dos elementos anatômicos da madeira de árvores de Eucalyptus grandis Hill ex Maiden aos sete anos. Revista Instituto Florestal, São Paulo, v. 16, n. 1, p. 43-55, 2004.

SENFT, J. F.; BENDTESEN, B. A.; GALLIGAN, W. L. Weak wood. Journal of Forestry, Bethesda, n. 83, p. 476-485, 1985.

SETTE JUNIOR, C. R. et al. Efeito da idade e posição de amostragem na densidade e características anatômicas da madeira de Eucalyptus grandis. Revista Árvore, Viçosa, MG, v. 36, n. 6, p. 1183-1190, 2012.

SHIMOYAMA, V. R. S. Variações da densidade básica e características anatômicas e químicas da madeira em Eucalyptus sp. 1990. 93 f. Dissertação (Mestrado em Engenharia Florestal) - Escola Superior de Agricultura "Luiz de Queiroz", Piracicaba, 1990.

SILVA, J. C. et al. Influência de idade e da posição radial nas dimensões das fibras e dos vasos da madeira de Eucalyptus grandis Hill ex Maiden. Revista Árvore, Viçosa, MG, v. 31, n. 6, p. 1081-1090, 2007.

SILVA, J. C. Influência dos tratamentos silviculturais na qualidade da madeira. Revista da Madeira, Curitiba, v. 10, n. 55, p. 62-65, 2001.

SILVA, J. R. M. Relações da usinabilidade e aderência do verniz com as propriedades fundamentais do Eucalyptus grandis Hill ex Maiden. 2002. 179 f. Tese (Doutorado em Engenharia Florestal) - Universidade Federal do Paraná, Curitiba, 2002.

TOMAZELLO FILHO, M. Variação radial da densidade básica e da estrutura anatômica da madeira de Eucalyptus saligna e Eucalyptus grandis. Revista IPEF, Piracicaba, v. 29, p. 37-45, 1985.

TOMAZELLO FILHO, M. Variação radial da densidade básica e da estrutura anatômica da madeira do Eucalyptus globulus, E. pellita e E. acmenioides. Revista IPEF, Piracicaba, n. 36, p. 35-42, 1987.

TREVISAN, R. et al. Efeito do desbaste no comprimento das fibras da madeira de Eucalyptus grandis W. Hill ex Maiden. Ciência Florestal, Santa Maria, v. 23, n. 2, p. 461-473, 2013.

VEENIN, T. et al. Radial variations of anatomical characteristics and specific gravity in Eucalyptus camadulensis clones. Iawa Journal, Leiden. v. 26, n. 3, p. 353-361, 2005.

ZOBEL B. J. The changing quality of the world wood supply. Wood Science and Technology, New York, v. 18, n. 1, p. $1-17,1984$. 